\title{
Broadband Horizontal and Vertical Spatial Coherence Measurements
}

\author{
Timothy H. Ruppel*, Steve Stanic*, Guy V, Norton ${ }^{\dagger}$, Roger W. Meredith*, \\ Edgar T. Kennedy*, Ralph R. Goodman** and Marcia A. Wilson* \\ ${ }^{*}$ Code 7184, Naval Research Laboratory, Stennis Space Center, MS 39529 \\ ${ }^{\dagger}$ Code 7181, Naval Research Laboratory, Stennis Space Center, MS 39529 \\ ${ }^{* *}$ Department of Marine Sciences, University of Southern Mississippi, Stennis Space Center, MS \\ 39529
}

\begin{abstract}
Initial results of broadband (1 to $10 \mathrm{kHz}$ ) spatial coherence measurements taken during the June 2003 shallow-water $(8 \mathrm{~m})$ propagation experiments will be presented. The results will show spatial coherence estimates over a $12 \mathrm{~m}$ long horizontal array and over a $6 \mathrm{~m}$ vertical array. The data was taken over a range of sea states and at ranges of approximately 70 and $150 \mathrm{~m}$.
\end{abstract}

\section{INTRODUCTION}

Shallow-water horizontal and vertical coherence measurements were taken during a series of experiments in June, 2003 off the coast of Panama City, FL. This paper reports initial results of these measurements at $70.4 \mathrm{~m}$ range. One set of measurements was taken during calm surface conditions, and one during the rough surface conditions that accompanied numerous thunderstorms. A number of other measurements were made over several days. Analysis of that data is ongoing. A brief summary of the important environmental parameters will be presented, followed by the horizontal and vertical coherence measurement results. Finally, conclusions will be drawn and our continuing analysis outlined.

\section{ENVIRONMENT}

A detailed description of the experimental procedure and the measured environment are found in other papers presented at this conference.[1, 2] In particular, Ref. [1] includes a brief survey of the previous work in this field. To summarize the procedure, the experiment took place in $8.8 \mathrm{~m}$ of water with a sandy bottom. A broadband source was mounted to a tower such that the source was $5.8 \mathrm{~m}$ below the surface. A $12 \mathrm{~m}$ horizontal array of eight unequally-spaced hydrophones was mounted on a separate tower $70 \mathrm{~m}$ away from the source tower. The horizontal array was mounted $7.1 \mathrm{~m}$ below the surface. A vertical array of ten hydrophones with a $0.53 \mathrm{~m}$ spacing was also mounted on the same tower as the horizontal array. Acoustic pings were transmitted once per second. The calm sea run was conducted on June 17 with winds at 5 knots under clear 


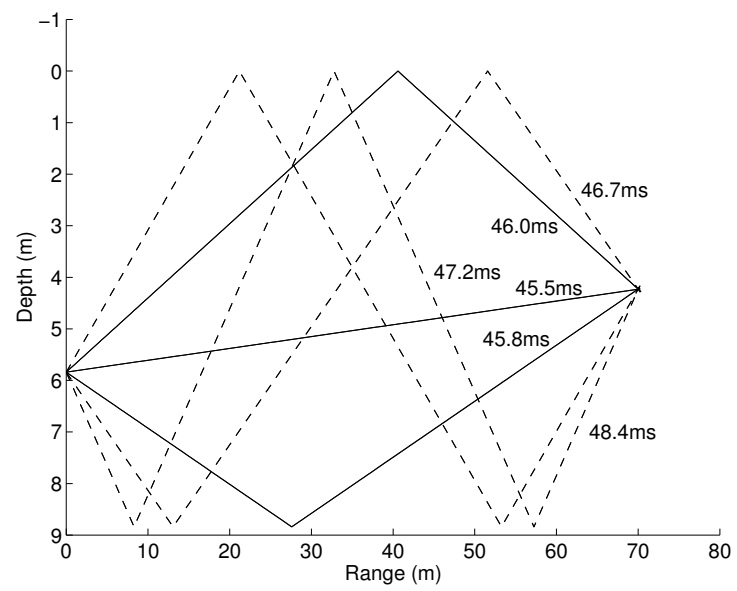

(a)

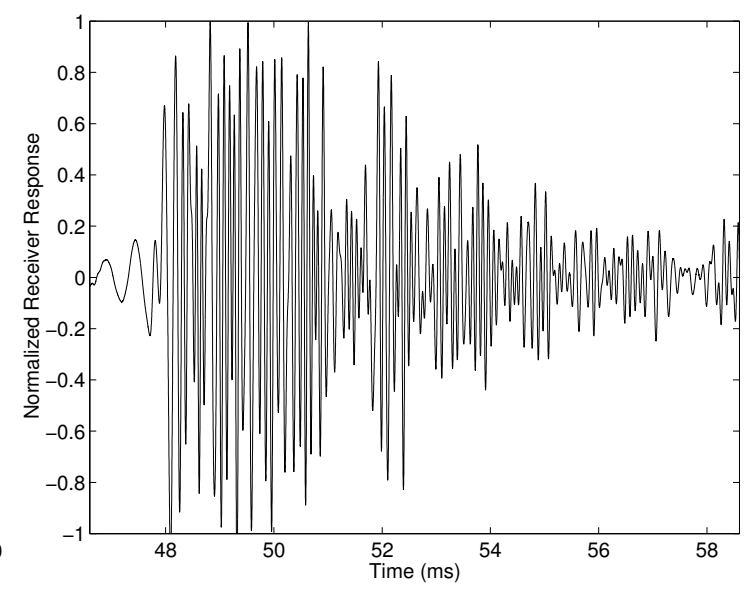

(b)

FIGURE 1. Ray traces from the source to one of the transducers on the vertical array assuming a constant $1539 \mathrm{~m} / \mathrm{s}$ sound speed in the water channel (a), and a sample receiver response for the calm sea run (b). The values shown on the ray trace are the arrival times for each ray.

conditions. The rough sea run was conducted on June 20 with winds at 13-17 knots during numerous thunderstorms.

Ray traces for an iso-velocity sound profile (measured at $1539 \mathrm{~m} / \mathrm{s}$ ), a sandy bottom, and smooth surfaces are shown in Fig. 1(a). Only the first few rays are shown. Plotted with each ray is its calculated arrival time. The shallow water and close range result in arrivals which are difficult to separate with only fractions of a millisecond separating them. The bottom-reflected ray arrives only $0.3 \mathrm{~ms}$ later than the direct ray, and the surface-reflected ray arrives only $0.2 \mathrm{~ms}$ after that. Fig. 1(b) shows the receiver response as a function of time for one of the hydrophones on the horizontal array during the calm sea run. A very complex multi-path arrival structure is evident in the signal.

\section{HORIZONTAL COHERENCE MEASUREMENTS}

Fig. 2 shows a time history and histogram of the horizontal coherence between two hydrophones $2.41 \mathrm{~m}$ apart during the calm sea run. Note the trend toward decreasing coherence (and increasing variation) with frequency. Fig. 3 shows the results of similar calculations for the rough sea run. As one might expect, the horizontal coherence measured is smaller, and the variation in those measurements is larger in the rough sea case. Fig. 4 shows the variation of the mean and standard deviation of the coherence between these hydrophones with each frequency calculated. The general downward trend in coherence is seen here as well, and is somewhat more pronounced in the rough sea data. Also seen in Fig. 4 are the large coherence variations. Fig. 5 summarizes the values for all the hydrophones in the array. Plotted are the mean values and standard deviations of coherence measured throughout the run for the frequencies shown in Fig. 2. Note the more rapid reduction of coherence with distance in the rough sea case, but the variations are quite large even for the calm sea run. 


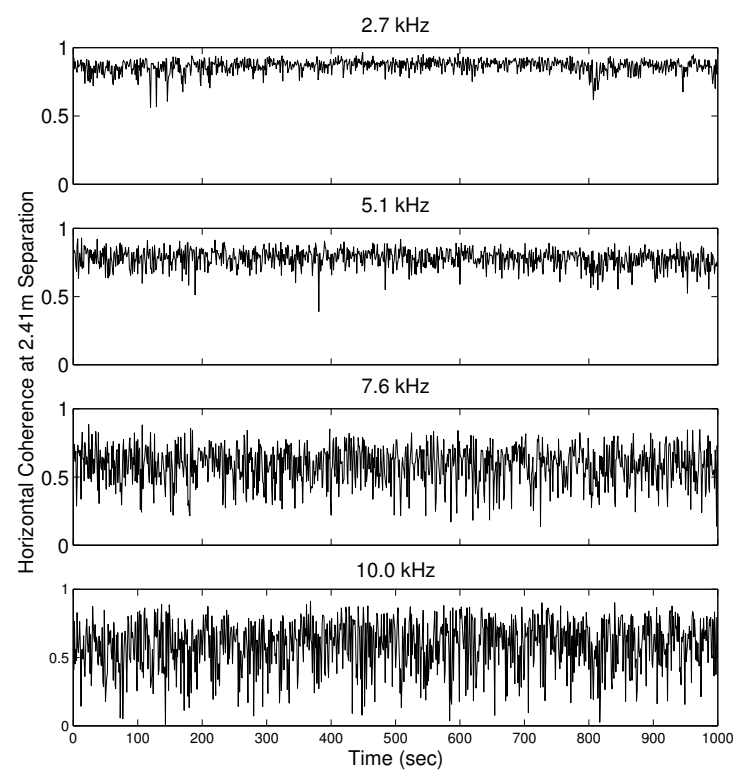

(a)

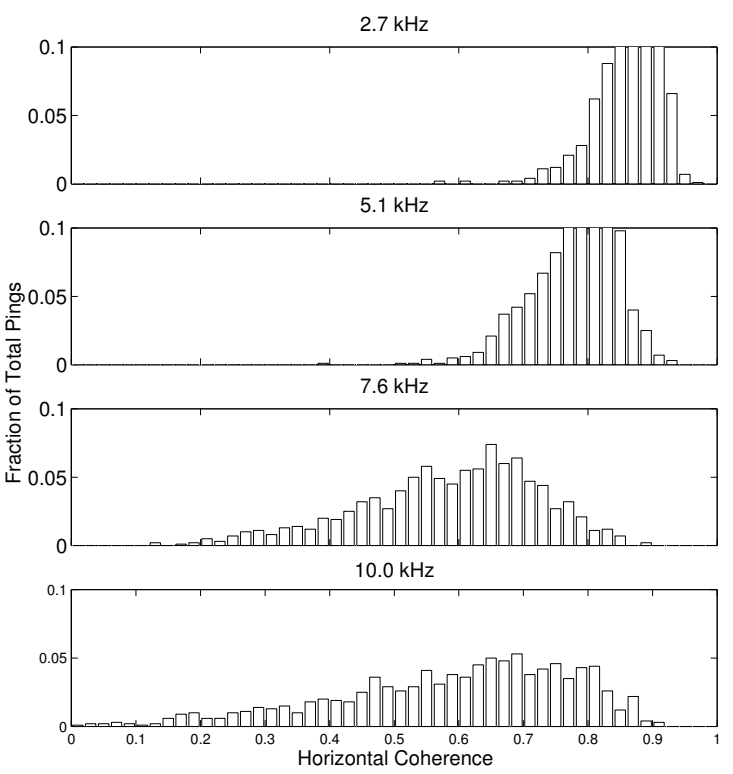

(b)

FIGURE 2. The measured horizontal coherence vs. time for four frequencies during the calm sea run (a), and a histogram of that data (b).

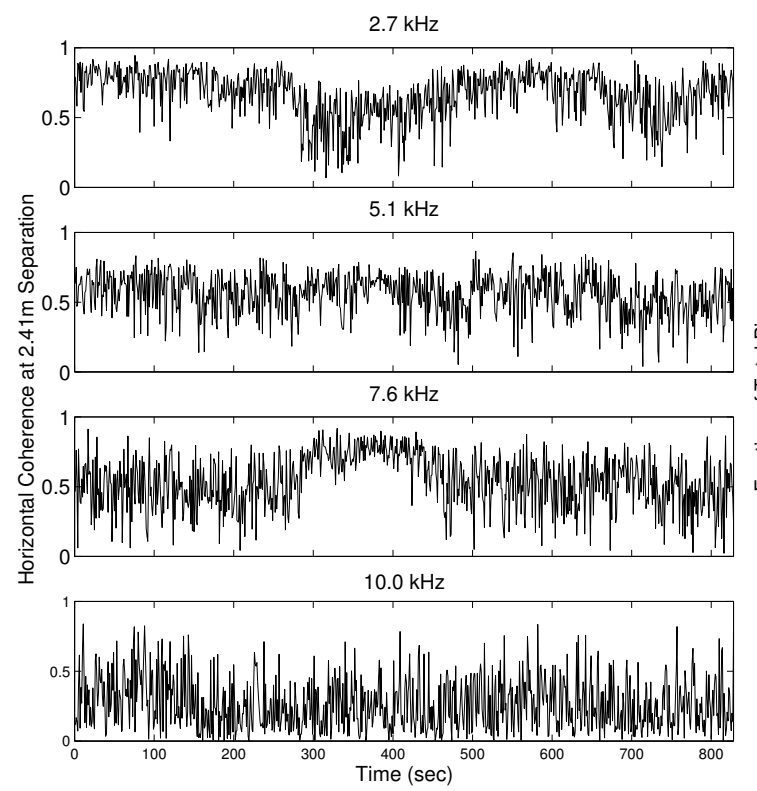

(a)

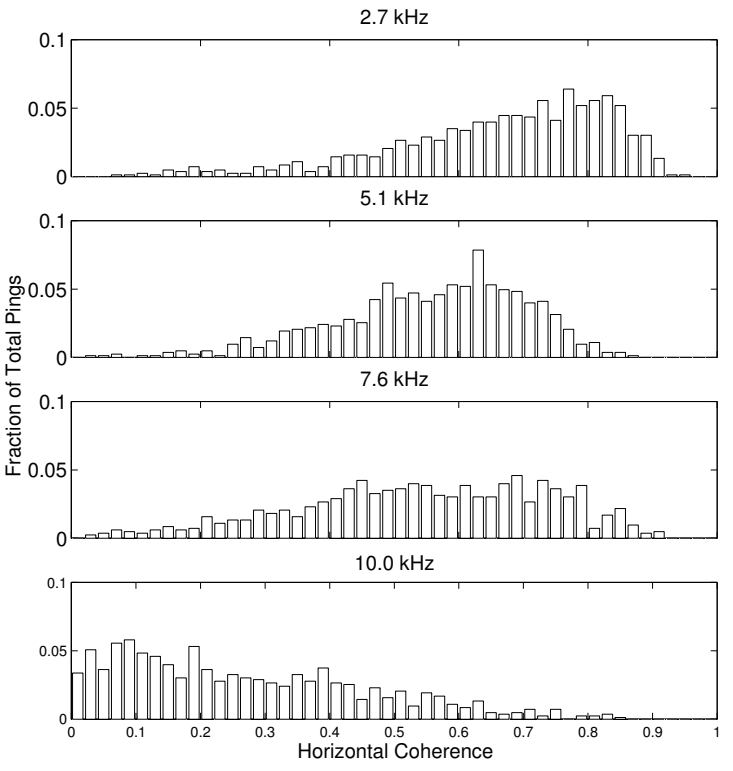

(b)

FIGURE 3. The horizontal coherence vs. time for four frequencies during the rough sea run (a), and a histogram of that data (b). 


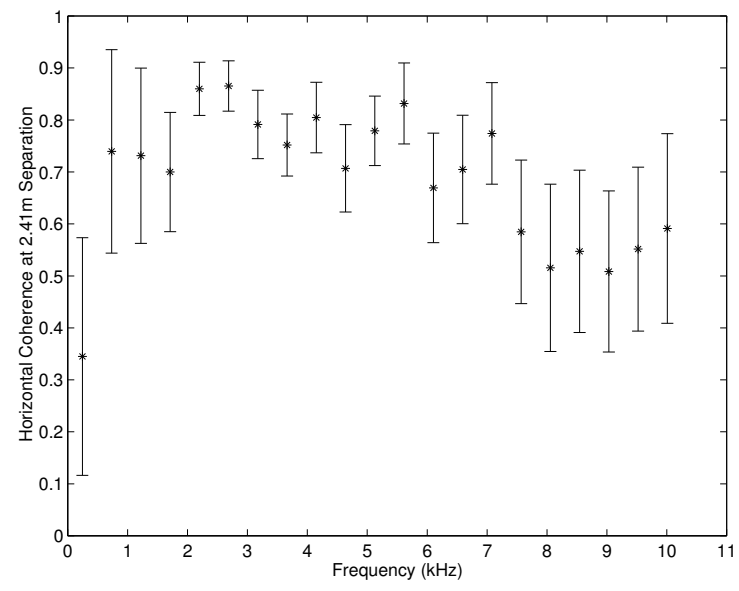

(a)

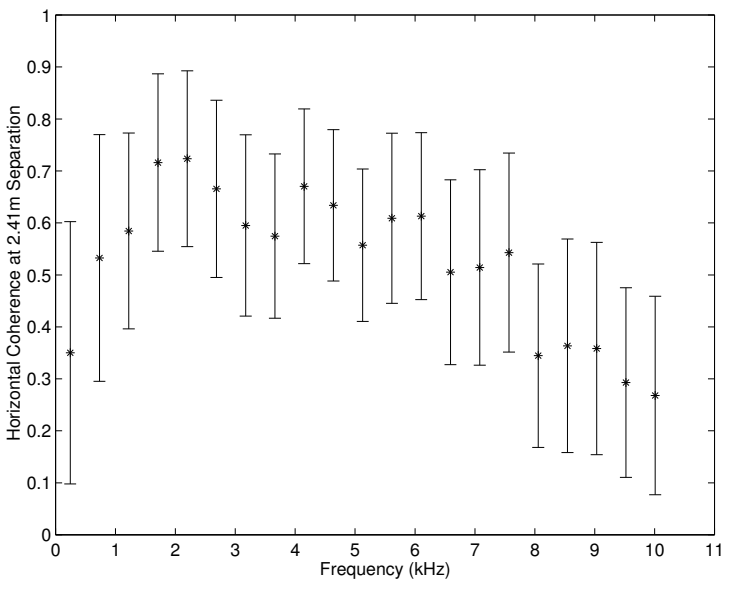

(b)

FIGURE 4. Frequency variation of mean and standard deviation of horizontal coherence measurements between two hydrophones $2.41 \mathrm{~m}$ apart during the calm sea (a) and rough sea (b) runs.
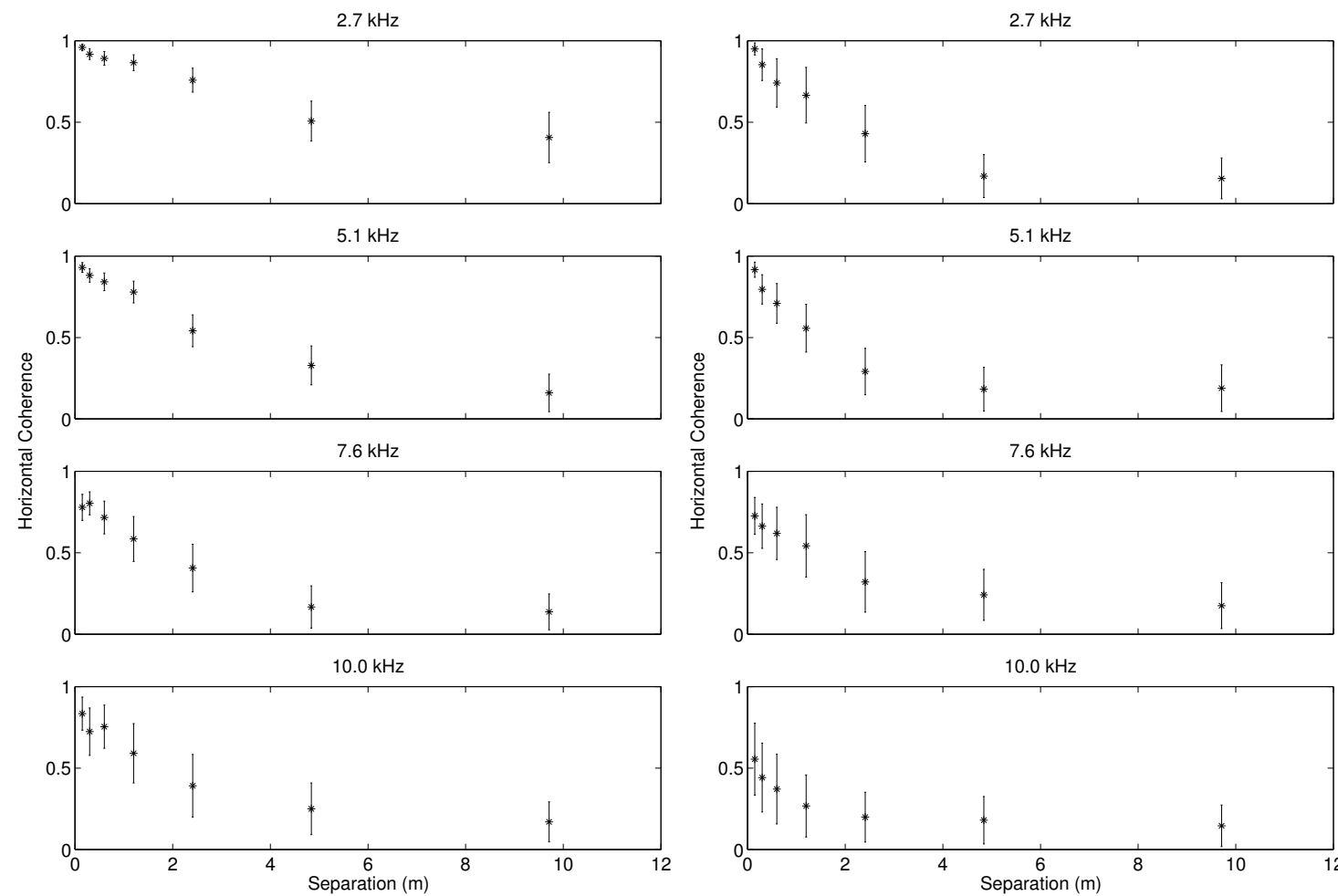

(a)

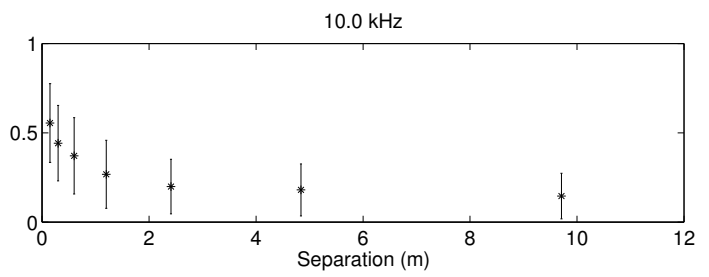

(b)

FIGURE 5. Variation of mean and standard deviation of horizontal coherence measurements with distance during the calm sea (a) and rough sea (b) runs. 


\section{VERTICAL COHERENCE MEASUREMENTS}

Fig. 6 shows a time history and histogram of the vertical coherence between two hydrophones $2.12 \mathrm{~m}$ apart during the calm sea run. Here the large variation appears in all frequency bins, though coherence seems to increase at $10 \mathrm{kHz}$. Also plotted in Fig. 6 are the results of a numerical model for an ideal flat sea with an iso-velocity sound speed profile. While the numerical model also predicts some increase in coherence at this frequency, it greatly underestimates the observed values. Fig. 7 shows analogous results for the rough sea run, again showing small vertical coherence, and large variation in that coherence. Note that the increased correlation at $10 \mathrm{kHz}$ is not as evident here. Fig. 8 shows the variation of the mean and standard deviation of the coherence between these hydrophones with each frequency calculated. Again, the ideal flat sea numerical predictions are plotted with the calm sea data in Fig. 8(a). Note that the mean values for the coherence do not vary as much with frequency in the rough sea data. Also note that the numerical model predicts a peak in coherence at about $9 \mathrm{kHz}$, so that the higher measured value at $10 \mathrm{kHz}$ may be due to the slightly rough surface. Fig. 9 summarize the values for all the hydrophones in the array. It can be seen that the variation in vertical coherence is quite large for both the calm sea and rough sea runs, and for each hydrophone.
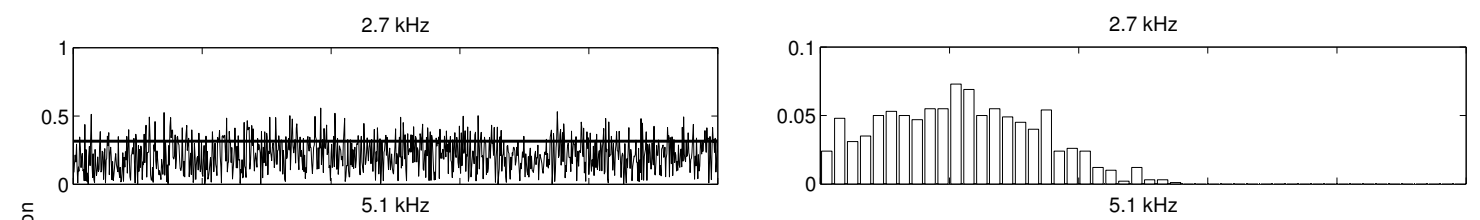

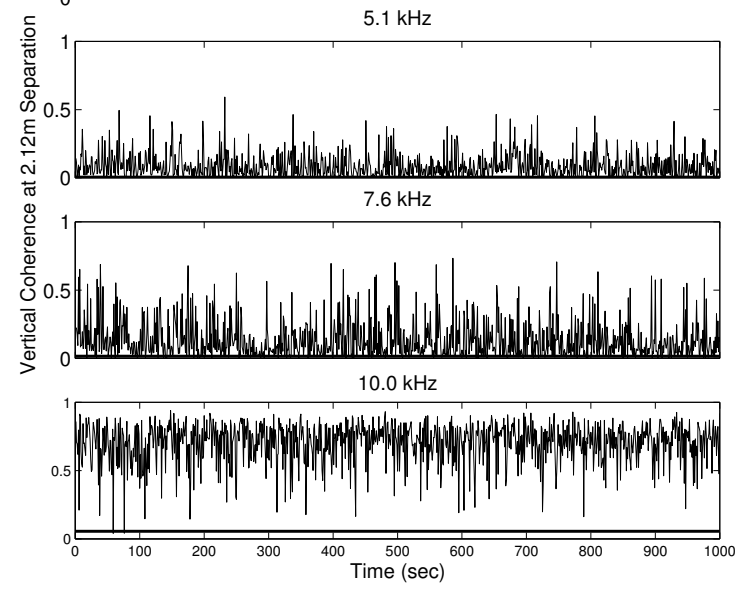

(a)

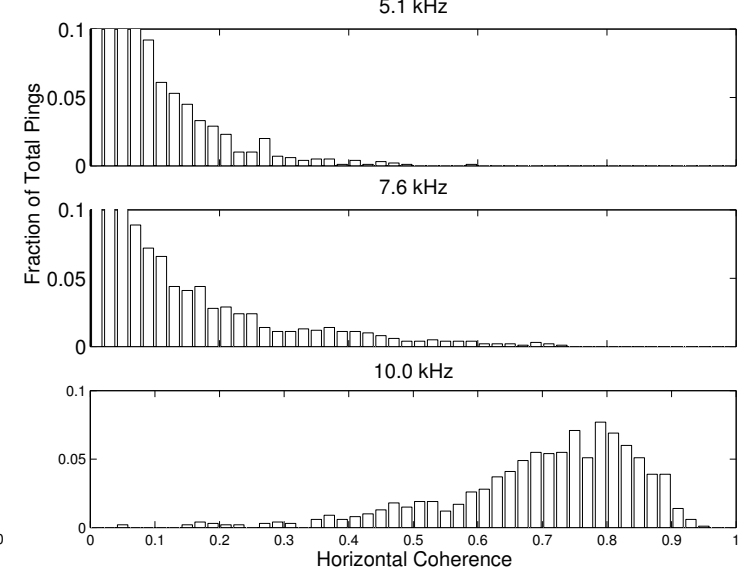

(b)

FIGURE 6. Vertical coherence vs. time for four frequencies during the calm sea run (a), and a histogram of that data (b). Also plotted are the results from an numerical model of an ideal flat surface, shown as a solid line. 
$2.7 \mathrm{kHz}$

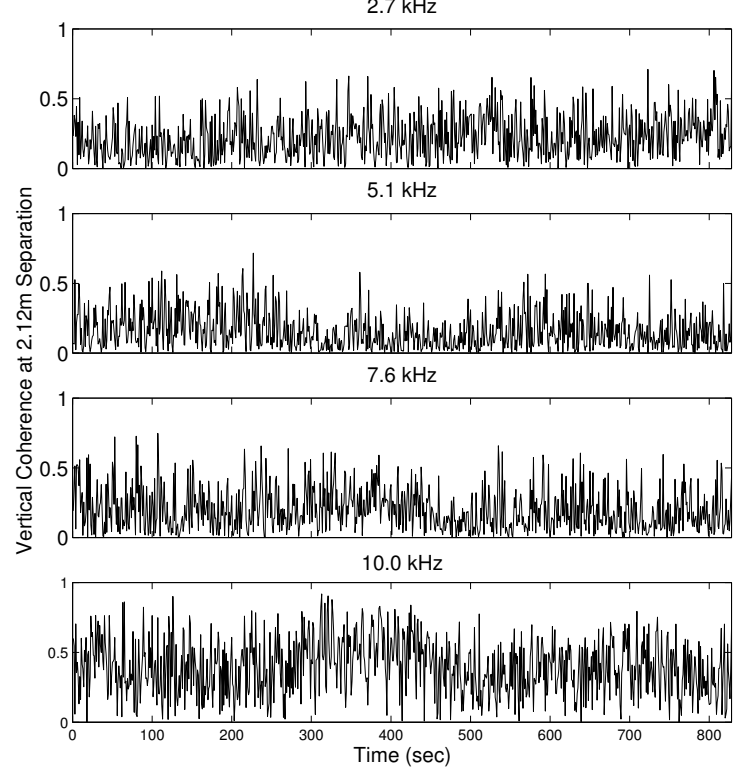

$2.7 \mathrm{kHz}$

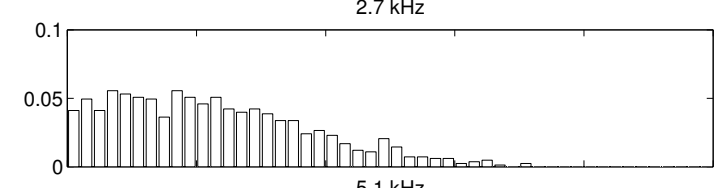

$5.1 \mathrm{kHz}$

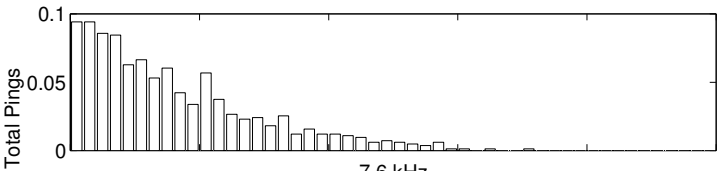

$7.6 \mathrm{kHz}$

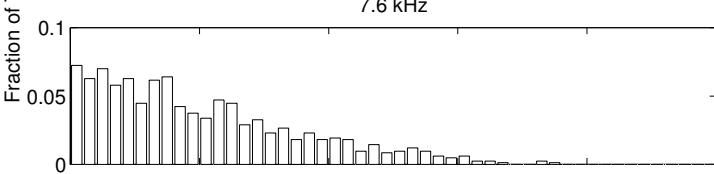

$10.0 \mathrm{kHz}$

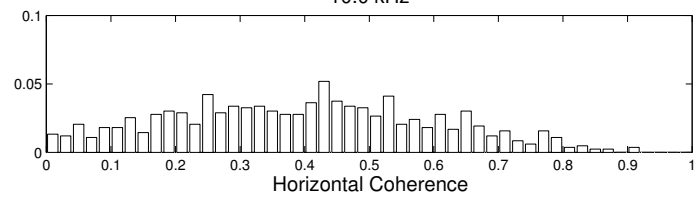

FIGURE 7. Vertical coherence vs. time for four frequencies during the rough sea run (a), and a histogram of that data (b).
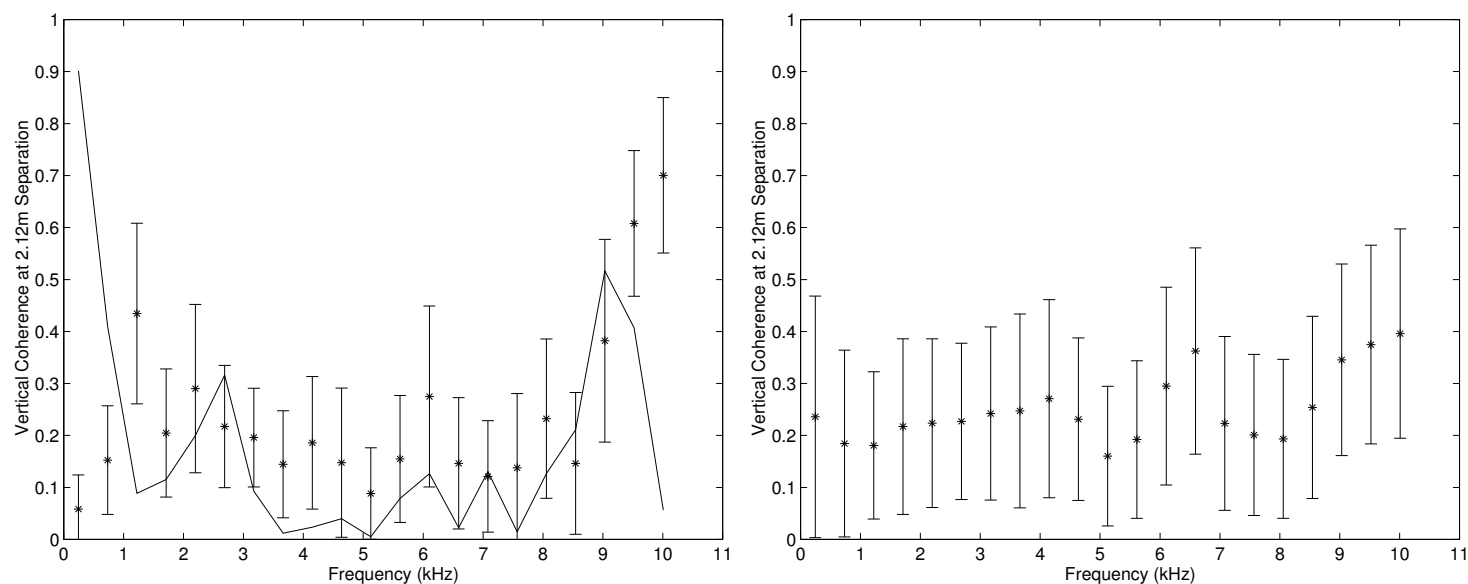

FIGURE 8. Frequency variation of mean and standard deviation of vertical coherence measurements between two hydrophones $2.12 \mathrm{~m}$ apart during the calm sea (a) and rough sea (b) runs. Numerical model results for an ideal flat-surface environment are plotted as a solid line with the calm sea data (a). 

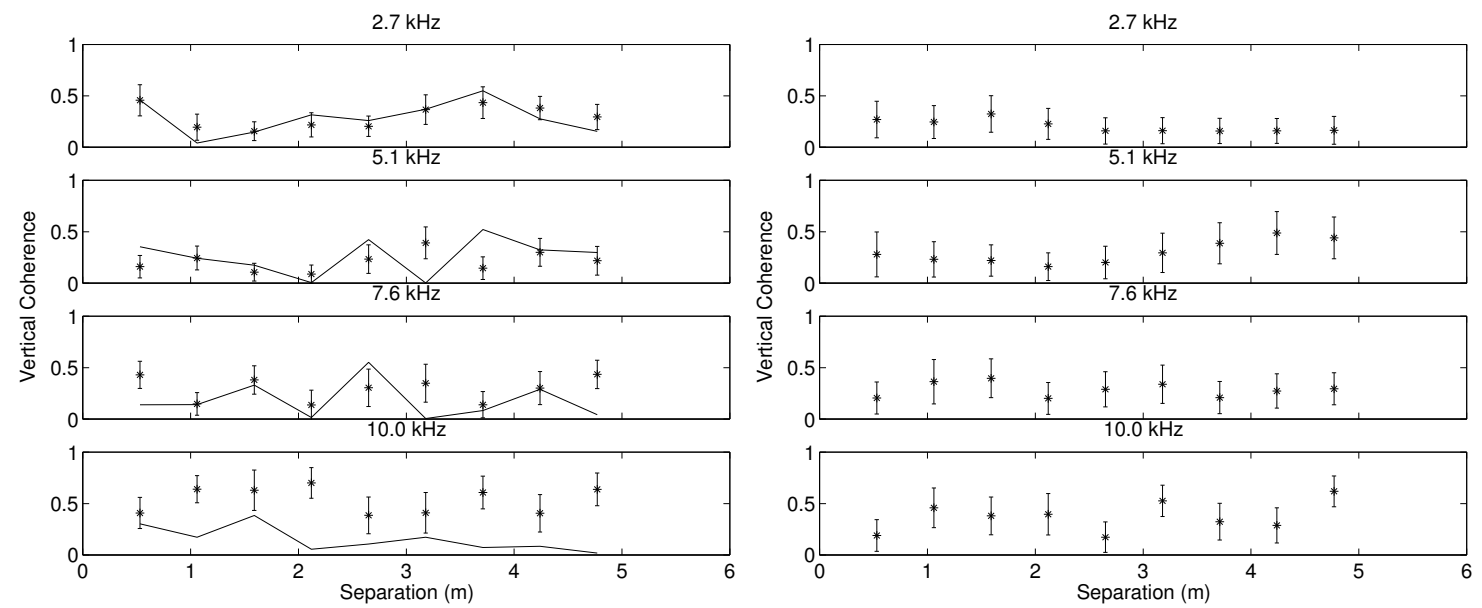

FIGURE 9. Variation of mean and standard deviation of vertical coherence measurements with distance during the calm sea (a) and rough sea (b) runs.

\section{CONCLUSIONS}

Preliminary results concerning the measured horizontal and vertical coherence from the June 2003 Panama City experiment have been presented. It has been shown that for $1-10 \mathrm{kHz}$ significant variations in coherence have been observed, even for fairly calms seas. Larger variations, particularly in horizontal coherence, are evident in rough seas. Measurements from different surface conditions and at the more distant source range have yet to be analyzed, and further analysis of all the data is planned.

\section{ACKNOWLEDGMENTS}

This work was supported by the Office of Naval Research, technical management by the Naval Research Laboratory under program element 62435N.

\section{REFERENCES}

1. Stanic, S., et al., "Panama City 2003 Broadband Shallow-Water Acoustic Coherence Experiments," in Proceedings of the High-Frequency Ocean Acoustics Conference, 2004.

2. Meredith, R., et al., "Panama City 2003 Acoustic Coherence Experiments: Environmental Characterization," in Proceedings of the High-Frequency Ocean Acoustics Conference, 2004. 